\title{
Palatabilité de onze fourrages tropicaux chez des cobayes (Cavia porcellus) de différents stades physiologiques au Bénin
}

\author{
Alex Ghêliho Zoffoun ${ }^{1,2^{*}}$, Abossèdé Murielle Lucrèce Faïhun ${ }^{3}$

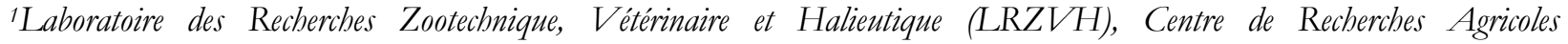 \\ d'Agonkanmey (CRA-Agonkanmey), Institut National des Recherches Agricoles du Bénin, (INRAB), 01 BP 2359 RP, \\ Cotonou, République du Bénin. \\ 2Laboratoire d'Ecologie Appliquée (LEA), Facultés des Sciences Agronomiques, Université d'Abomey-Calavi (UAC), 01 BP \\ 526. Cotonou - Bénin. \\ ${ }^{3}$ Laboratoire d'Ethnopharmacologie et de Santé Animale, Faculté des Sciences Agronomiques, Université d'Abomey-Calavi \\ (UAC) Bénin. \\ * Auteur correspondant : email : alex.zoffoun35@gmail.com Tél:00 22996697153
}

Mots clés : Palatabilité, fourrage, cobaye, Bénin.

Keywords: Palatability, fodder, guinea pig, Benin.

Publication date 31/08/2019, http://www.m.elewa.org/JAPS

\section{RESUME}

Evaluer la palatabilité de onze fourrages tropicaux chez les cobayes (Cavia porcellus).

Des cobayes appartenant à deux stades physiologiques (jeunes et adultes) ont fait l'objet des tests sur la palatabilité. Onze espèces fourragères tirées des résultats d'enquête auprès des éleveurs locaux ont été utilisées : Panicum maximum C1(Herbe de Guinée var C1), Panicum maximum local (Herbe de Guinée local), Pennisetum purpureum (Herbe éléphant), Elaeis guineensis (Palmier à huile), Musa acuminata (Bananier), Manihot esculenta (Manioc), Carica papaya (Papayer), Centrosema pubescens (Haricot sauvage), Moringa olifera (Moringe), Talinum triangulare (Pourpier tropical) et Tridax procumbens (Herbe à lapins). L'indice de palatabilité (IP) de chaque fourrage a été déterminé selon la méthode décrite par Salem et al. (2000). Panicum maximum local a présenté l'indice de préférence le plus élevé aussi bien chez les jeunes que chez les adultes, les valeurs ont été respectivement de 0,22 et 0,38. Centrosoma pubescens, Tridax procumbens et Panicum maximum $C 1$ ont aussi présenté des indices de préférence élevés pour les deux stades physiologiques des cobayes. Les valeurs obtenues pour Centrosoma pubescens ont été de 0,22 chez les jeunes contre 0,25 chez les adultes, pour Tridax procumbens 0,19 chez les jeunes contre 0,24 chez les adultes, pour Panicum maximum C1 0,10 chez les jeunes contre 0,15 chez les adultes. Les valeurs d'indice de préférence les plus faibles ont été obtenues pour les espèces fourragères Musa acuminata $(I P=0,00 \mathrm{chez}$ les jeunes contre $0,02 \mathrm{chez}$ les adultes) et Elaeis guineensis ( $I P=0,01 \mathrm{chez}$ les jeunes contre 0,01 chez les adultes). Les valeurs obtenues pour les indices de préférences ont été influencées significativement par l'âge de l'animal ( $\mathrm{p}$-value $=0.005651)$ et le type de fourrage $(\mathrm{p}$-value $<2.2 \mathrm{e}-16)$. La présente étude a permis d'évaluer la palatabilité de onze espèces fourragères chez des cobayes de différents stades physiologiques et d'en retenir les plus appétées par ces animaux. La connaissance des espèces fourragères les plus appréciées par les cobayes et la composition chimique de ces dernières permettront d'optimiser les stratégies d'alimentation dans les élevages. 
Palatability of eleven tropical forages in guinea pigs (Cavia porcellus) of different physiological stages in Benin

To evaluate the palatability of eleven tropical forages in guinea pigs (Cavia porcellus).

Guinea pigs from two physiological stages (young and adult) were tested for palatability. Eleven forage species from survey results from local breeders were used : Panicum maximum C1 (Guinea grass C1), Panicum maximum local (local Guinea grass), Pennisetum purpureum (Elephant grass), Elaeis guineensis (African oil palm), Musa acuminata (Banana), Manihot esculenta (Cassava), Carica papaya (Pawpaw), Centrosema pubescens (Butterfly pea), Moringa olifera (Moringa), Talinum triangulare (Potherb fameflower) et Tridax procumbens (Coat buttons). The palatability index (PI) of each forage was determined by following the procedure described by Salem et al. (2000). Local Panicum maximum had the highest preference index in both young and adults, with values of 0.22 and 0.38, respectively. Centrosoma pubescens, Tridax procumbens and Panicum maximum $\mathrm{C1}$ also showed high preference indices for the two physiological stages of guinea pigs. The values obtained for Centrosoma pubescens were 0.22 in the young against 0.25 in the adults, for Tridax procumbens 0.19 in the young against 0.24 in the adults, for Panicum maximum $\mathrm{C} 1,0.10$ in the young against 0.15 in adults. The lowest preference index values were obtained for the forage species Musa acuminata (IP $=0.00$ in young versus 0.02 in adults) and Elaeis guineensis (IP $=0.01$ in young versus 0.01 in adults). The values obtained for the preference indices were significantly influenced by the age of the animal ( $p$-value = $0.005651)$ and the type of forage ( $\mathrm{p}$-value $<2.2 \mathrm{e}-16)$. The present study evaluated the palatability of eleven forage species in guinea pigs of different physiological stages and retained the most palatable of these animals. The knowledge of the forage species most appreciated by the guinea pigs and the chemical composition of the latter will optimize the feeding strategies in the farms.

\section{INTRODUCTION}

La compréhension des relations plante-animal, à travers le comportement alimentaire et l'ingestion, sont des connaissances nécessaires pour une gestion rationnelle des parcours et des troupeaux (Zoffoun et al., 2011). Elle permet de déceler les déséquilibres des écosystèmes pâturés, de juger de l'adéquation entre les ressources fourragères et la population animale qui les exploite, de raisonner la complémentation en fonction des carences réelles du régime (Guérin, 1988) mais aussi et surtout de prédire les performances animales (Orsini, 1991). Les animaux d'une façon générale ont la capacité de choisir l'aliment à consommer. Ainsi plusieurs mécanismes comportementaux (état de faim, apprentissage social, néophobie, etc.) participent à la sélection et à la composition de la ration (Dumont et al., 2001). En Afrique tropicale, l'alimentation représente le principal facteur limitant l'expression du potentiel de production des animaux d'élevage comme les cobayes (Tendonkeng et al., 2011). L'alimentation des animaux est constituée de quantités inadéquates et de la mauvaise qualité des aliments (Ondiek et al., 2017). Les ressources fourragères locales sont sous-utilisées, car la ration quotidienne des animaux est souvent à base d'une seule espèce fourragère supplémentée parfois par des déchets de cuisine pauvres en éléments nutritifs. La connaissance des espèces fourragères les plus appréciées par les cobayes et la composition chimique de ces dernières permettront d'optimiser les stratégies d'alimentation dans les élevages. Ainsi, des cobayes appartenant à deux stades physiologiques ont fait l'objet des tests sur la palatabilité. 


\section{MÉTHODOLOGIE}

3.1 Matériel animal et dispositif expérimental: Deux lots de six cobayes chacun ont été utilisés: le premier lot de 3 mâles $(245,19 \pm 65,41 \mathrm{~g})$ et 3 femelles $(251,78 \pm 51,35 \mathrm{~g})$ âgés de deux mois environ et le deuxième lot de 3 mâles $(441,05 \pm 48,26 \mathrm{~g})$ et 3 femelles $(446,80 \pm 56,08 \mathrm{~g})$ âgés de six mois environ. Les animaux ont été achetés dans les fermes privées du département du Mono au Bénin. A leur arrivée à la Ferme d'application dans la Commune d'Abomey-Calavi au Sud Bénin, les cobayes ont été logés par groupe de sexe dans des loges en briques de 100 x 100 x $90 \mathrm{~cm}$ et au fond cimenté. La période de mise en quarantaine a duré deux semaines, l'alimentation était composée de Panicum maximum et de son de blé. L'eau a été également servie dans les abreuvoirs ad libitum.

3.2 Matériel végétal : Onze espèces fourragères tirées des résultats d'enquête auprès des éleveurs locaux ont été utilisées. Ce sont : Panicum maximum C1, Panicum maximum local, Pennisetum purpureum (coupé à une hauteur de 30 $\mathrm{cm})$, Elaeis guineensis (feuilles), Musa acuminata, Manihot esculenta, Carica papaya (feuilles séchées à l'ombre la veille), Centrosema pubescens (feuilles et tiges molles), Moringa olifera, Talinum triangulare et Tridax procumbens. Toutes les espèces fourragères étudiées à l'exception de Manihot esculenta, et de Musa acuminata étaient au stade de fructification. La composition chimique des différents fourrages est présentée dans le tableau 1.

Tableau 1 : Composition chimique des différents fourrages testés

\begin{tabular}{l|l|l|l|l|l}
\hline \multirow{2}{*}{ Espèces fourragères } & \multicolumn{4}{l}{ Composition chimique (\%MS) } \\
\cline { 2 - 6 } & MS & MG & PB & MM & CB \\
\hline Panicum maximum C1 & 95,8 & 2,5 & 19,11 & 9,89 & 22,89 \\
\hline Panicum maximum local & 95,87 & 2,4 & 19,11 & 9,81 & 22,83 \\
\hline Musa acuminata & 91,78 & 1,70 & 17,3 & 19 & 28,8 \\
\hline Elaeis guineensis & 94,9 & 5,00 & 4,2 & 5,6 & 48,1 \\
\hline Pennisetum purpureum & 94,5 & 2,5 & 8,88 & 7,71 & 31,52 \\
\hline Centrosema pubescens & 94,63 & 3,14 & 23 & 7,72 & 32 \\
\hline Moringa olifera & 18,7 & 6,5 & 29 & 12 & 19,1 \\
\hline Manibot esculenta & 94,38 & 1,3 & 22 & 9,89 & 15 \\
\hline Tridax procumbens & 95,91 & 4,92 & 12,82 & 14,00 & 20 \\
\hline Carica papaya & 91,73 & 2 & 21 & 2,2 & 1,8 \\
\hline Talinum triangulare & 94,31 & 0,4 & 3,6 & 24,68 & 4,4 \\
\hline MS: & & &
\end{tabular}

MS : Matière Sèche ; MG : Matières Grasses ; PB : Protéines Brutes ; MM : Matières Minérales ; CB : Cendres Brutes.

Sources : Lebas, 1996 ; Saka et al., 2008 ; Adjolohoun et al., 2013 ; Noumbissi et al., 2014 ; Bengaly, 2015 ; Kouakou et al., 2015 ; Koukoui et al., 2017

3.3 Conduite de l'essai et collecte des données

3.3.1 Phase d'adaptation aux espèces fourragères à étudier: $\mathrm{Au}$ cours de cette phase tous les fourrages à étudier ont été servis tous en même temps aux animaux à l'exception de Panicum maximum local qui constituait le fourrage de base des animaux lors de leur acquisition. Cette phase a duré une semaine et les échantillons de fourrages ont été renouvelés dans les loges tous les deux jours pour permettre aux animaux d'être en contact avec toutes les espèces fourragères.

3.3.2 Evaluation de l'appétence des espèces fourragères : Au cours de cette phase, les fourrages ont été divisés en deux groupe (fourrages riches en protéines et fourrages riches en fibres) Ainsi le premier groupe de 
fourrages testés était composé de Panicum maximum C1, Panicum maximum local, Musa acuminata, Elaeis guineensis et Pennisetum purpureum; le second groupe de fourrages testés était composé de Centrosema pubescens, Moringa olifera, Manibot esculenta, Tridax procumbens, Carica papaya et Talinum triangulare. Le test a été réalisé en deux étapes respectivement suivant les deux groupes de fourrage. Les cages ayant servis à la conduite du test étaient en briques de 100 x 100 x $90 \mathrm{~cm}$ et au fond cimenté. Une quantité de 20 $\mathrm{g}$ de chaque fourrage a été pesée et servie dans des mangeoires disposées de façon aléatoire dans la loge. Chaque animal a été par la suite introduit pour une durée de $30 \mathrm{mn}$ dans chacune des cages équipées des mangeoires contenant les espèces fourragères. Cette opération a été répétée deux fois au cours de la journée, le matin à 8 heures et le soir à 15 heures. Après 30 minutes de contact des animaux aux espèces fourragères, ces animaux ont été sortis des cages et les différents refus pesés. L'alimentation des animaux en dehors des moments du test était composée de son de blé uniquement afin de susciter l'envie de fourrage chez eux. La collecte des données a duré 9jours, les différents échantillons des espèces fourragères ont été récoltées chaque jour et préfanées à température ambiante avant utilisation. Les animaux ont été pesés au début et à la fin du test, la pesée a été effectuée le matin avant le service d'un nouveau repas. La consommation de fourrage a été évaluée par la différence entre la quantité servie et le refus.

3.4 Paramètres calculés et analyse de données : Les différents paramètres calculés sont: quantité de chaque fourrage consommée par l'animal le matin au cours du test de palatabilité (Q1), quantité de chaque fourrage consommée par l'animal le soir au cours du test de palatabilité (Q2), l'indice de palatabilité de chaque fourrage (IP) selon la méthode décrite par Salem et al. (2000).

$\mathrm{Q} 1(\mathrm{~g})=20 \mathrm{~g}$ - refus

$\mathrm{Q} 2(\mathrm{~g})=20 \mathrm{~g}-$ refus

$\mathrm{IP}=\mathrm{C} 9 / \mathrm{O} 9$

Avec $20 \mathrm{~g}=$ quantité de matières fraîches offertes au cours du test de palatabilité ; C9 = quantité moyenne de fourrage consommée quotidiennement au cours du test de palatabilité; $\mathrm{O} 9=$ quantité moyenne de fourrage offerte au cours du test de palatabilité.

Les données de consommation moyenne et d'indice de préférence ont été soumises au test non paramétrique de Kruskall Wallis, les différences ont été considérées significatives au seuil de 5\%. Les facteurs étudiés sont le sexe et l'âge des animaux, le type de fourrage, le moment de déroulement du test (matin et soir). 


\section{RÉSULTATS}

Palatabilité des différentes espèces fourrages

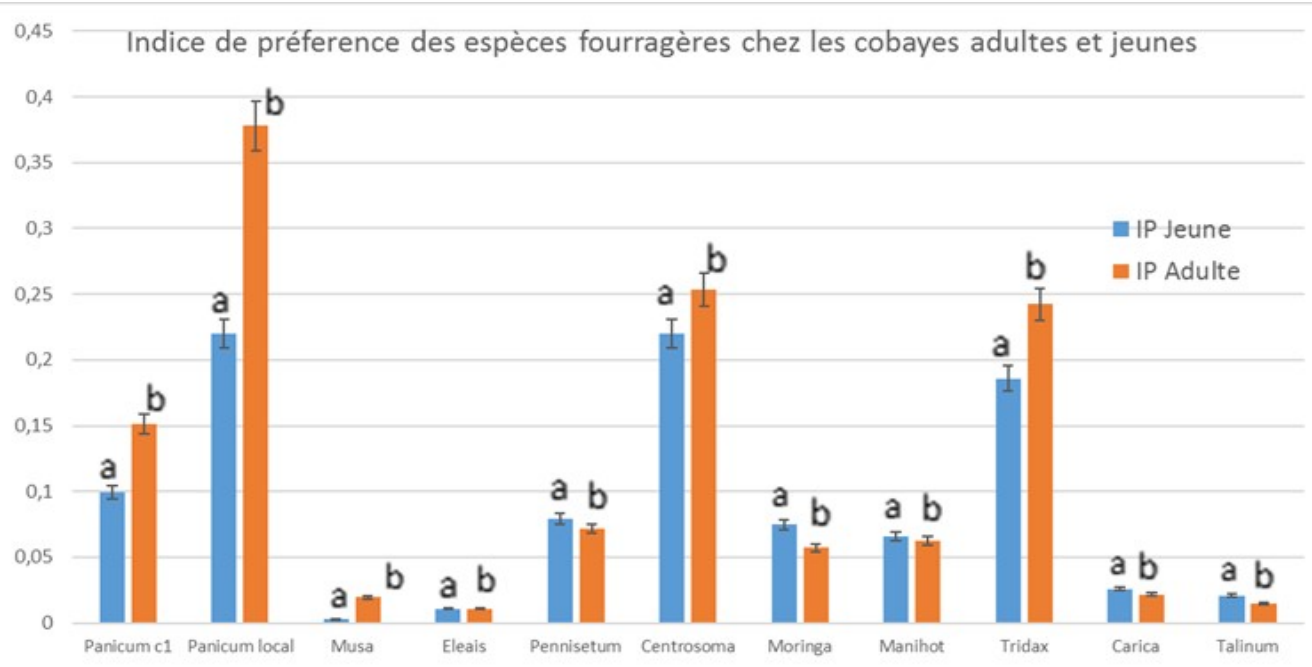

Figure 1 : Comparaison des indices de préférence des différentes espèces fourragères chez les jeunes cobayes et les cobayes adultes

Panicum maximum local a présenté l'indice de préférence le plus élevé aussi bien chez les jeunes que chez les adultes, les valeurs ont été respectivement de 0,22 et 0,38. Centrosoma pubescens, Tridax procumbens et Panicum maximum C1 ont aussi présenté des indices de préférence élevés pour les deux stades physiologiques des cobayes. Les valeurs obtenues pour Centrosoma pubescens ont été de 0,22 chez les jeunes contre 0,25 chez les adultes, pour Tridax procumbens 0,19 chez les jeunes contre 0,24 chez les adultes, pour Panicum maximum C1 0,10 chez les jeunes contre 0,15 chez les adultes. Les valeurs d'indice de préférence les plus faibles ont été obtenues pour les espèces fourragères Musa acuminata (IP $=0,00$ chez les jeunes contre 0,02 chez les adultes) et Elaeis guineensis (IP $=0,01$ chez les jeunes contre 0,01 chez les adultes) (Figure 1). Les valeurs obtenues pour les indices de préférences ont été influencées significativement par l'âge de l'animal ( $\mathrm{p}$-value $=0.005651)$ et le type de fourrage ( $\mathrm{p}$-value $<$ 2.2e-16). Lorsqu'on considère le même stade physiologique (cobayes jeunes ou cobayes adultes), le sexe n'a pas influencé les indices de préférences $(p$-value $=0.9734)$. Chez les jeunes, les femelles ont eu un indice de préférence plus élevé pour Panicum maximum local (IP $=0,29)$, Centrosoma pubescens $(0,22)$, Tridax procumbens $(0,17)$; les mâles ont eu comme premiers choix Centrosoma pubescens $(\mathrm{IP}=0,23)$ et Tridax procumbens $(\mathrm{IP}=0,23)$ suivi de Panicum maximum local (IP=0,21). Musa acuminata et Elaeis guineensis demeurent les espèces les moins appréciées par les animaux de ce stade physiologique (Tableau 1). Chez les adultes, les femelles ont plus préféré Panicum maximum local $(\mathrm{IP}=0,35)$, Tridax procumbens $(\mathrm{IP}=0,28)$ et Centrosoma pubescens $(0,22)$; les mâles ont plutôt préféré Panicum maximum local ( $\mathrm{IP}=0,41)$, Centrosoma pubescens $(\mathrm{IP}=0,29)$ et Tridax procumbens $(0,20)$. Les espèces fourragères les moins appétées par les animaux de ce stade physiologique sont Musa acuminata, Elaeis guineensis, Carica papaya et Talinum triangulare (Tableau 2). La période c'est dire le moment de déroulement du test (matin ou soir) a influencé significativement l'indice de préférence des fourrages $(p$-value $=0.003148)$. Les espèces fourragères riches en protéines ont été plus consommées le matin se traduisant par des valeurs d'indice de préférence plus élevées (Tableau 2) et (Tableau 3). 
Tableau 2 : Consommation moyenne et indice de palatabilité des différents fourrages chez les jeunes cobayes

\begin{tabular}{|c|c|c|c|c|c|c|c|}
\hline Sexe & Fourrages & Q1(g) & $\mathbf{I P}_{1}$ & Q2(g) & $\mathbf{I P}_{2}$ & C9(g) & IP \\
\hline \multirow[t]{11}{*}{ Femelle } & Panicum maximum C1 & $1,52 \pm 0,95$ & 0,07 & $1,80 \pm 0,74$ & 0,09 & $1,70 \pm 0,2$ & 0,09 \\
\hline & Panicum maximum local & $4,12 \pm 0,86$ & 0,21 & $4,74 \pm 0,87$ & 0,24 & $4,53 \pm 0,44$ & 0,23 \\
\hline & Musa acuminata & $0,01 \pm 0,02$ & 0,00 & $0,03 \pm 0,06$ & 0,00 & $0,02 \pm 0,02$ & 0,00 \\
\hline & Elaeis guineensis & $0,18 \pm 0,25$ & 0,009 & $0,12 \pm 0,18$ & 0,006 & $0,14 \pm 0,05$ & 0,01 \\
\hline & Pennisetum purpureum & $2,14 \pm 1,28$ & 0,11 & $1,72 \pm 1,15$ & 0,09 & $1,86 \pm 0,29$ & 0,09 \\
\hline & Centrosema pubescens & $4,73 \pm 1,85$ & 0,24 & $4,05 \pm 1,16$ & 0,20 & $4,39 \pm 0,24$ & 0,22 \\
\hline & Moringa olifera & $1,72 \pm 2,30$ & 0,09 & $0,89 \pm 0,60$ & 0,04 & $1,31 \pm 0,29$ & 0,07 \\
\hline & Manibot esculenta & $1,70 \pm 1,54$ & 0,09 & $1,14 \pm 0,61$ & 0,06 & $1,42 \pm 0,2$ & 0,07 \\
\hline & Tridax procumbens & $3,73 \pm 2,96$ & 0,19 & $3,23 \pm 1,40$ & 0,16 & $3,48 \pm 0,18$ & 0,17 \\
\hline & Carica papaya & $0,81 \pm 1,74$ & 0,04 & $0,31 \pm 0,49$ & 0,02 & $0,56 \pm 0,18$ & 0,03 \\
\hline & Talinum triangulare & $0,65 \pm 0,92$ & 0,03 & $0,15 \pm 0,23$ & 0,01 & $0,40 \pm 0,18$ & 0,02 \\
\hline \multirow[t]{11}{*}{ Mâle } & Panicum maximum C1 & $1,38 \pm 1,38$ & 0,07 & $3,14 \pm 1,13$ & 0,16 & $2,26 \pm 1,25$ & 0,11 \\
\hline & Panicum maximum local & $4,63 \pm 1,53$ & 0,23 & $3,92 \pm 1,18$ & 0,20 & $4,28 \pm 0,50$ & 0,21 \\
\hline & Musa acuminata & $0,12 \pm 0,14$ & 0,01 & $0,09 \pm 0,17$ & 0,00 & $0,11 \pm 0,02$ & 0,01 \\
\hline & Elaeis guineensis & $0,38 \pm 0,29$ & 0,02 & $0,21 \pm 0,23$ & 0,01 & $0,29 \pm 0,12$ & 0,01 \\
\hline & Pennisetum purpureum & $1,38 \pm 0,80$ & 0,07 & $1,23 \pm 0,57$ & 0,06 & $1,30 \pm 0,11$ & 0,07 \\
\hline & Centrosema pubescens & $4,63 \pm 0,24$ & 0,23 & $4,17 \pm 1,00$ & 0,21 & $4,40 \pm 0,33$ & 0,23 \\
\hline & Moringa olifera & $2,20 \pm 0,29$ & 0,11 & $1,16 \pm 0,68$ & 0,05 & $1,68 \pm 0,74$ & 0,11 \\
\hline & Manibot esculenta & $1,35 \pm 0,20$ & 0,07 & $1,09 \pm 0,54$ & 0.05 & $1,22 \pm 0,18$ & 0,07 \\
\hline & Tridax procumbens & $4,56 \pm 0,18$ & 0,23 & $3,30 \pm 1,50$ & 0,20 & $3,96 \pm 0,85$ & 0,23 \\
\hline & Carica papaya & $0,68 \pm 0,18$ & 0,03 & $0,27 \pm 0,31$ & 0,01 & $0,47 \pm 0,29$ & 0,03 \\
\hline & Talinum triangulare & $0,76 \pm 0,18$ & 0,04 & $0,12 \pm 0,17$ & 0,00 & $0,44 \pm 0,45$ & 0,04 \\
\hline
\end{tabular}

Tableau 3: Consommation moyenne et indice de palatabilité des différents fourrages chez les cobayes adultes

\begin{tabular}{|c|c|c|c|c|c|c|c|}
\hline Sexe & Fourrages & Q1(g) & IPmat & Q2(g) & IPsoir & C9(g) & IP \\
\hline \multirow[t]{11}{*}{ Femelle } & Panicum maximum C1 & $3,19 \pm 1,85$ & 0,16 & $3,13 \pm 1,14$ & 0,16 & $3,16 \pm 0,04$ & 0,16 \\
\hline & Panicum maximum local & $7,07 \pm 2,59$ & 0,35 & $6,94 \pm 2,17$ & 0,35 & $7,00 \pm 0,09$ & 0,35 \\
\hline & Musa acuminata & $0,20 \pm 0,23$ & 0,01 & $0,33 \pm 0,50$ & 0,02 & $0,26 \pm 0,09$ & 0,01 \\
\hline & Elaeis guineensis & $0,26 \pm 0,20$ & 0,01 & $0,39 \pm 0,44$ & 0,02 & $0,33 \pm 0,09$ & 0,02 \\
\hline & Pennisetum purpureum & $1,48 \pm 0,62$ & 0,07 & $1,33 \pm 1,23$ & 0,07 & $1,41 \pm 0,11$ & 0,07 \\
\hline & Centrosema pubescens & $3,88 \pm 1,27$ & 0,20 & $4,80 \pm 1,04$ & 0,24 & $4,34 \pm 0,65$ & 0,22 \\
\hline & Moringa olifera & $1,37 \pm 0,80$ & 0,07 & $1,02 \pm 0,36$ & 0,05 & $1,20 \pm 0,24$ & 0,06 \\
\hline & Manibot esculenta & $1,25 \pm 0,71$ & 0,06 & $1,46 \pm 0,26$ & 0,07 & $1,36 \pm 0,15$ & 0,07 \\
\hline & Tridax procumbens & $5,24 \pm 1,02$ & 0,26 & $6,07 \pm 1,71$ & 0,30 & $5,65 \pm 0,59$ & 0,28 \\
\hline & Carica papaya & $0,75 \pm 0,59$ & 0,04 & $0,41 \pm 0,33$ & 0,02 & $0,58 \pm 0,24$ & 0,03 \\
\hline & Talinum triangulare & $0,18 \pm 0,37$ & 0,01 & $0,04 \pm 0,06$ & 0,00 & $0,11 \pm 0,10$ & 0,01 \\
\hline \multirow[t]{11}{*}{ Mâle } & Panicum maximum C1 & $2,51 \pm 0,04$ & 0,13 & $3,24 \pm 1,14$ & 0,16 & $2,87 \pm 0,51$ & 0,14 \\
\hline & Panicum maximum local & $8,68 \pm 0,09$ & 0,43 & $7,57 \pm 1,94$ & 0,38 & $8,13 \pm 0,79$ & 0,41 \\
\hline & Musa acuminata & $0,49 \pm 0,09$ & 0,02 & $0,54 \pm 0,93$ & 0,03 & $0,51 \pm 0,04$ & 0,03 \\
\hline & Elaeis guineensis & $0,13 \pm 0,09$ & 0,01 & $0,10 \pm 0,14$ & 0,01 & $0,11 \pm 0,02$ & 0,01 \\
\hline & Pennisetum purpureum & $1,66 \pm 0,11$ & 0,08 & $1,27 \pm 0,60$ & 0,06 & $1,47 \pm 0,28$ & 0,07 \\
\hline & Centrosema pubescens & $5,84 \pm 1,57$ & 0,29 & $5,75 \pm 0,50$ & 0,29 & $5,80 \pm 0,07$ & 0,29 \\
\hline & Moringa olifera & $1,26 \pm 0,31$ & 0,06 & $0,92 \pm 0,52$ & 0,05 & $1,09 \pm 0,25$ & 0,05 \\
\hline & Manibot esculenta & $1,32 \pm 0,77$ & 0,07 & $0,97 \pm 0,39$ & 0,05 & $1,14 \pm 0,25$ & 0,06 \\
\hline & Tridax procumbens & $4,02 \pm 1,49$ & 0,20 & $4,07 \pm 1,68$ & 0,20 & $4,05 \pm 0,03$ & 0,20 \\
\hline & Carica papaya & $0,25 \pm 0,25$ & 0,01 & $0,32 \pm 0,38$ & 0,02 & $0,29 \pm 0,05$ & 0,01 \\
\hline & Talinum triangulare & $0,73 \pm 0,54$ & 0,04 & $0,28 \pm 0,36$ & 0,014 & $0,49 \pm 0,33$ & 0,02 \\
\hline
\end{tabular}


Un classement des espèces suivant leurs indices de préférence nous permet de retenir cet ordre décroissant de préférence: Panicum maximum local $>$ Centrosoma pubescencens $>$ Tridax procumbescens $>$ Panicum maximum $C 1>$ Penisetum

\section{$5 \quad$ DISCUSSION}

La palatabilité des différents fourrages étudiés a varié significativement en fonction de l'âge des animaux et du type d'espèces fourragères. Le premier choix des animaux a été Panicum maximum local suivi par ordre de grandeur de Centrosoma pubescens et Tridax procumbens. La palatabilité ou l'appétibilité en effet désigne les caractéristiques de l'aliment qui provoquent la réaction des sens de l'animal et donc un appétit plus ou moins développé pour cet aliment (Dumont, 1996). Elle dépend d'une part de l'expérience, de l'état métabolique et physiologique de l'animal (Dumont, 1995; Forbes, 1995) et d'autre part des caractéristiques physiques et chimiques de l'aliment (Jarrige, 1988). La préférence des animaux des deux stades physiologiques considérés (jeune et adulte) au Panicum maximum local serait liée à leur expérience alimentaire dans les exploitations privées d'origine, les éleveurs nourrissant la plupart du temps les animaux avec cette espèce fourragère. Les herbivores acquièrent des préférences alimentaires qui résultent de l'apprentissage avec la mère et d'expérience dans le jeune âge. Un jeune animal nourrit par une espèce fourragère pendant une courte période avant ou pendant le sevrage, choisit préférentiellement par la suite l'espèce qu'il connait déjà (Ramos et Tennessen, 1992). De plus les expériences alimentaires dans le jeune âge influencent plus les choix des animaux que leurs expériences ultérieures (Arnold et Maller, 1977). Une préférence élevée pour Centrosoma pubescens et Tridax procumbens par les cobayes de cette étude pourrait s'expliquer par la bonne composition chimique et le bon goût de ces fourrages. Les choix alimentaires des herbivores sont avant tout dictés par la recherche de sensations purpureum $>$ Moringa oléifera $>$ Manibot esculenta $>$ Carica papaya $>$ Talinum triangulare $>$ Elaeis guineensis $>$ Musa acuminata.

agréables, gustatives, olfactives et tactiles ; ces choix alimentaires seront surtout orientés par le goût (Arnold, 1987). La dépréciation notée pour les fourrages tels que Musa acuminata et Elaeis guineensis serait liée à la qualité des éléments nutritifs qu'ils referment ou à la présence de facteurs anti-nutritionnels. En effet, les herbivores acquièrent des aversions alimentaires pour des aliments associés à des composés toxiques et limitent l'ingestion de régimes trop riches ou au contraire déficients en énergie ou en certains nutriments (Zahorik et al., 1990 ; Nicholson et al., 1992 ; Provenza, 1995 ; Zoffoun et al., 2011). Les différents indices de préférence obtenus dans cette étude sont nettement en dessous de ceux obtenus par Kampemba et al. (2017) ; cette différence dans les valeurs obtenues réside dans la durée de contact (24 heures) des cobayes avec les fourrages dans cet essai. Ces mêmes auteurs ont rapporté que Penisetum purpureum a un indice de préférence plus élevé que Panicum maximum ce qui pousse à penser que le milieu de vie ou d'élevage des cobayes et même le fourrage de base pourraient influencer leurs habitudes alimentaires. Moringa oléifera encore appelé « arbre de vie » par les profanes n'est pas du tout apprécié à l'état frais par les cobayes. Ses feuilles contiennent des saponines qui donnent un goût amer lors de l'ingestion par les animaux (Makkar et al., 1997) ce qui a sans doute repoussé les cobayes. Il est donc opportun d'envisager son incorporation dans des rations alimentaires granuleuses comme l'indiquent les travaux de Tedonkeng Pamo et al. (2005). Nouman et al. (2014) ont montré que l'addition de Moringa oleifera dans la ration est beaucoup plus bénéfique à cause de la présence des acides 
gras dans les feuilles ce qui améliorent la palatabilité.

\section{CONCLUSION}

La présente étude a permis d'évaluer la palatabilité de onze espèces fourragères chez des cobayes de différents stades physiologiques. Quatre espèces fourragères sur les onze ont présenté des indices de préférence élevés pour les deux stades physiologiques des cobayes: Panicum maximum local, Centrosoma pubescens, Tridax procumbens et Panicum maximum C1. La

\section{RÉFÉRENCES BIBLIOGRAPHIQUES}

Adjolohoun S., Dahouda M., Adandedjan C., TolebaS. S., Kindomihou V. \& Sinsin B: 2013. Evaluation of biomass production and nutritive value of nine Panicum maximum ecotypes in Central region of Benin, African Journal of Agricultural Research, 8(17), 1661-1668, DOI: 10.5897/AJAR 12.2026

Arnold G.W. \& Maller R. A: 1977. Effects of nutritional experience in early and adult life on the performance and dietary habits of sheep. Appl. Anim. Ethol., 3, 5-26.

Arnold G.W: 1987. Influence of the biomass, botanical composition and sward height of annual pastures on foraging behaviour by sheep. J. Appl. Ecol., 24, 759-772.

Bengaly K. : 2015. Supplémentation de feuilles de palmier à huile (Elaeis guineensis) traitées à la vapeur à des agneaux en Malaisie, Tropicultura, 33,1, 46-54

Dumont B.: 1995. Déterminisme des choix alimentaires des herbivores au pâturage : principales théories. INRA Productions animales, 8 (4), 285-292.

Dumont B.: 1996. Préférences et sélection alimentaire au pâturage, INRA Prod. Anim., 9 (5), 359-366.

Dumont B., Meuret M., Boissy A. \& Petit M. : 2001. Le pâturage vu par l'animal: mécanismes comportementaux et application en élevage, Fourrages 166, 213-238. palatabilité des fourrages n'a pas été influencé par le sexe de l'animal mais par l'âge de ce dernier et le type de fourrage. La connaissance des espèces fourragères les plus appréciées par les cobayes et la composition chimique de ces dernières permettront d'optimiser les stratégies d'alimentation dans les élevages.

Forbes J. M. : 1995. Voluntary food intake and diet selection in farm animals. $\mathrm{CAB}$ International, Wallingford, Oxon, UK, $532 \mathrm{p}$.

Guerin H.: 1988. Régime alimentaire de ruminants domestiques (bovins, ovins, caprins) exploitant des parcours naturels sahéliens et soudano-sahéliens. Rappels bibliographiques sur les objectifs et les méthodes d'étude de la composition botanique des régimes ingérés au pâturage. Revue d'Elevage et de Médecine vétérinaire des Pays tropicaux. 41 : 419-426.

Jarrige R.: 1988. Alimentation des bovins, ovins et caprins. INRA, Paris, $471 \mathrm{p}$.

Kampemba F.M., Tshibangu M.I., Nyongombe N.U. \& Hornick J-L.: 2017. Palatability of nine fodders species used by guinea pigs (Cavia porcellus), Trop Anim Health Prod, DOI 10.1007/s11250017-1386-5.

Kouakou N.D.V., Kouakou N.J.A., Iritie B. M., Adjiadjemian S.B., Diarrassouba Z., N'Guessan K. R. \& Kouba M.: 2015. Effet de l'herbe de Guinée (Panicum maximum Jacq.) associée à l'herbe de lait (Euphorbia heterophylla L.) ou aux feuilles de patate douce (Ipomoea batatas L.) sur la croissance des lapins (Oryctolagus cuniculus L.), Journal of Applied Biosciences 93:8688 - 8695, http://dx.doi.org/10.4314/jab.v93i1.3

Koukoui O., Medegan S., Houngbèmè B., Mansoura B., Gbaguidi F. \& Sezan A. : 2017. Propriétés nutritionnelles et 
phytochimiques de Launaea taraxacifolia et Tridax procumbens, deux alicaments sous utilisés au Bénin, BRAB, ISSN 1025-2355 pp 11-19

Lebas F., Coudert P., Rochambeau H. \& Thébault R.G. : 1996. Le lapin : élevage et pathologie. FAO éditeur Rome, 227 pages

Makkar, H. P. S., Blümmel M., and Becker K.: 1997. Formation of complexes between polyvinily pyrorolidones or polyethylene glycols and their implication in gas production and true digestibility in vitro techniques. British Journal of Nutrition, 73, 897-913.

Nicholson J.W.G., Charmley E. \& Bush R.S., 1992. The effect of supplemental protein source on ammonia levels in rumen fluid and blood and intake of alfalfa silage by beef cattle. Can. J. Anim. Sci., 72, 853-862.

Noumbissi M.N.B., Tendonkeng F., Zougou T.G., and Tedonkeng Pamo E. : 2014. Effet de différents niveaux de supplémentation de feuilles de Tithonia diversifolia (Hemsl) A.Gray sur l'ingestion et la digestibilité in vivo de Pennisetum purpureum K. Schum. Chez le cobaye (Cavia porcellus L.), Tropicultura, 32, 3, 138-146.

Nouman, W., Basra, S. M. A., Siddiqui, M. T., Yasmeen, A., Gull, T., Alcayde \& M. A. C. : 2014. Potential of Moringa Oleifera L. as Livestock fodder crop: a review. Turkish Journal of Agriculture and Forestry, 38: 1-14.

Ondiek J O, Abdulrazak S A and Njoka E N.: 2017. Nutritive value and palatability rating of fifteen selected indigenous Kenyan browse species fed to Small East African goats, Livestock Research for Rural Development 29 (6), http:/ / www.lrrd.org/lrrd29/6/cont290 6.htm consulté le 20/04/2018.

Orsini J.-P. G.: 1991. Preference coefficients as index of selectivity in grazing ruminants. Actes du quatrième congrès international des terres de parcours, volume 2, Montpellier, France, pp. $653-$ 655.

Provenza F.D.: 1995. Postingestive feedback as an elementary determinant of food preference and intake in ruminants. J. Range Manage., 48, 2-17.

Ramos A. and Tennessen T.: 1992. Effect of previous grazing experience on the grazing behaviour of lambs. Appl. Anim. Behav. Sci., 33, 43-52.

Saka S., Munusamy, M.V., Shibata M., Tono Y. and Miyafuji H.: 2008. Chemical Constituents of the different anatomical parts of the oil palm (Elaeis guineensis) for their sustainable utilization, Seminar Proceedings, Natural Ressources and Energy Environment, (2),19-34

Salem H.B., Nefzaoui A. and Salem, L.B.: 2000. Sheep and goat preferences for Mediterranean fodder shrubs. Relationship with the nutritive characteristics. in Sheep and goat nutrition: Intake, digestion, quality of products and rangelands, CIHEAMAAMZ Zaragoza, Cahiers Options Méditerranéennes; 52, 155-159.

Tedonkeng Pamo E., Niba AT, Fonteh FA, Tendonkeng F, Kana JR and Boukila B: 2005. Effet de la supplémentation au Moringa oleifera ou aux blocs multinutritionnels sur l'évolution du poids post partum et la croissance présevrage des cobayes (Cavia porcellus L.). Livestock Research for rural Development. 2005.

Tendonkeng F, Boukila, Pamo T E, Mboko A V, Zogang F B and Matumuini N E F : 2011. Effets direct et résiduel de différents niveaux de fertilisation azotée sur la composition chimique de Brachiaria ruziziensis à la floraison à l'Ouest Cameroun. International Journal of Biological and Chemical Sciences 5 (2): 570-585.

Zahorik D.M., Houpt K.A. and SwartzmanAndert J.: 1990. Taste-aversion learning in three species of ruminants. Appl. Anim. Behav. Sci., 26, 27-39. 
Zoffoun, A. G., Babatounde, S., Houinato, M, Mensah, G. A. \& Sinsin, B.: 2011. Comportement alimentaire des taurillons Girolando sur deux types de pâturages cultivés en zone subéquatoriale. Canadian Journal of Animal Science $91:$ (4) 675 - 683. 